\title{
Application of Kalman Filter Model Based on Hyperbolic Curve Model in the Deformation forecast
}

\author{
Fumin $L u^{1, a}$ \\ ${ }^{1}$ Key Laboratory of Geological Hazards on Three Gorges Reservoir Area of Ministry of Education, \\ Three Gorges University, Yichang City, China \\ alfm640929@ctgu.edu.cn
}

Keywords: hyperbolic curve model; Kalman filter; dynamic noise; settlement; forecast

Abstract: The hyperbolic curve model is erected, the least square method is used to obtain parameters of the hyperbolic curve model, parameters of the hyperbolic curve model are regarded as state vectors to contain dynamic noises to erect Kalman filter model based on the hyperbolic curve model, on the basis of Kalman filter model based on the hyperbolic curve model, settlement amounts of the building are forecasted. Because parameters of Kalman filter model change continuously in the process of Kalman filter, the ability that Kalman filter model suit the observation data is increased, and the fitting error of the model is lessened. An example of calculation shows that the forecast error is small, and the forecast effect is better to use Kalman filter model based on the hyperbolic curve model to forecast settlement amounts of the building .

\section{Introduction}

In order to guarantee the safety of buildings, the settlement observation must be done termly to obtain the settlement observation data, on the basis of the settlement observation data, the forecast model that the fitting precision and forecast precision is high can be erected. The model parameters are looked as fixed values in the hyperbolic curve model, the ability that the model suit the observation data is decreased, the fitting forecast is big, the forecast effect is not very well. In order to raise the fitting precision and forecast effect, model parameters of hyperbolic curve model are looked as state vectors to contain dynamic noises, Kalman filter model based on hyperbolic curve model is erected to forecast settlement amounts of the building. An example of calculation shows that the forecast error is small, and the forecast effect is better.

\section{Hyperbolic curve model}

Hyperbolic curve model can be written as ${ }^{[1]}$

$$
\frac{t}{S_{t}-S_{0}}=a+b t
$$

Where $S_{0}$ is the initial settlement value of the building, $t$ is the observation time, $a$ and $b$ are model parameters of hyperbolic curve model, $S_{t}$ is the settlement value of the building at time $t$.

Let $y=\frac{t}{S_{t}-S_{0}}$, (1) can be written as

$$
y=a+b t
$$

On the basis of the settlement observation series, model parameters $a$ and $b$ can be obtained by means of least square method, the settlement value of the building can be forecasted.

\section{Kalman filter model}

The state equation and observation equation of the discrete linear system to Kalman filter model $\operatorname{are}^{[2]-[10]}$

$$
X_{k+1}=\Phi_{k+1, k} X_{k}+\Omega_{k}
$$




$$
L_{k+1}=B_{k+1} X_{k+1}+\Delta_{k+1}
$$

Where $X_{k}$ is the state vector at the time $t_{k}, L_{k}$ is the observation vector at the time $t_{k}, \Phi_{k+1, k}$ is the state transfer matrix at the time $t_{k}$ to $t_{k+1}, B_{k+1}$ is the observation matrix at the time $t_{k+1}, \Omega_{k}$ is the dynamic noise at the time $t_{k}, \Delta_{k}$ is the observation noise at the time $t_{k}$.

The random model of Kalman filter method are ${ }^{[2]-[10]}$

$$
\begin{aligned}
& E\left(\Omega_{k}\right)=0, E\left(\Delta_{k}\right)=0, \operatorname{cov}\left(\Omega_{k}, \Omega_{j}\right)=D_{\Omega}(k) \delta_{k j}, \operatorname{cov}\left(\Delta_{k}, \Delta_{j}\right)=D_{\Delta}(k) \delta_{k j}, \operatorname{cov}\left(\Omega_{k}, \Delta_{j}\right)=0, \\
& E\left(X_{0}\right)=\mu_{X}(0)=X(0 / 0), \operatorname{var}\left(X_{0}\right)=D_{X}(0), \operatorname{cov}\left(X_{0}, \Omega_{k}\right)=0, \operatorname{cov}\left(X_{0}, \Delta_{k}\right)=0 \\
& \quad \text { If } j=k, \delta_{k j}=1, \text { if } j \neq k, \delta_{k j}=0
\end{aligned}
$$

Where $E\left(\Omega_{k}\right)$ is the mathematical expectation of $\Omega_{k}, E\left(\Delta_{k}\right)$ is the mathematical expectation of $\Delta_{k}, \operatorname{cov}\left(\Omega_{k}, \Omega_{j}\right)$ is the covariance of $\Omega_{k}$ and $\Omega_{j}, D_{\Omega}(k)$ is the variance of $\Omega_{k}, \operatorname{cov}\left(\Delta_{k}, \Delta_{j}\right)$ is the covariance of $\Delta_{k}$ and $\Delta_{j}, D_{\Delta}(k)$ is the variance of $\Delta_{k}, \operatorname{cov}\left(\Omega_{k}, \Delta_{j}\right)$ is the covariance of $\Omega_{k}$ and $\Delta_{j}$, $E\left(X_{0}\right)$ is the mathematical expectation of $X_{0}, \operatorname{var}\left(X_{0}\right)$ is the variance of $X_{0}, \operatorname{cov}\left(X_{0}, \Omega_{k}\right)$ is the covariance of $X_{0}$ and $\Omega_{k}, \operatorname{cov}\left(X_{0}, \Delta_{k}\right)$ is the covariance of $X_{0}$ and $\Delta_{k}$.

On the basis of the state equation and observation equation and random model, Kalman filter equations are obtained ${ }^{[2]-[10]}$

$$
\begin{aligned}
X(k / k) & =X(k / k-1)+J_{k}\left[L_{k}-B_{k} X(k / k-1)\right] \\
D_{X}(k / k) & =\left[I-J_{k} B_{k}\right] D_{X}(k / k-1)
\end{aligned}
$$

Where $I$ is a unit matrix, and

$$
\begin{aligned}
X(k / k-1) & =\Phi_{k, k-1} X(k-1 / k-1) \\
D_{X}(k / k-1) & =\Phi_{k, k-1} D_{X}(k-1 / k-1) \Phi_{k, k-1}^{T}+D_{\Omega}(k-1) \\
J_{k} & =D_{X}(k / k-1) B_{k}^{T}\left[B_{k} D_{X}(k / k-1) B_{k}^{T}+D_{\Delta}(k)\right]^{-1}
\end{aligned}
$$

\section{Kalman filter model based on hyperbolic curve model}

In order to improve the fitting precision of hyperbolic curve model, model parameters of hyperbolic curve model $a$ and $b$ are looked as the state vector to contain dynamic noises, Kalman filter method is used to filter to obtain the best estimated value of the state vector, the settlement value of the building can be forecasted, we can erect the following model

$$
y_{k}=a+b t_{k}+\Delta_{k}
$$

Where $a$ and $b$ are model parameters of hyperbolic curve model, $t_{k}$ is the observation time, $\Delta_{k}$ is the observation noise at the observation time $t_{k}, y_{k}=\frac{t_{k}}{S_{t_{k}}-S_{0}}$.

Let $L_{k}=y_{k}, B_{k}=\left[\begin{array}{ll}1 & t_{k}\end{array}\right], X_{k}=\left[\begin{array}{l}a \\ b\end{array}\right],(8)$ can be written as

$$
L_{k}=B_{k} X_{k}+\Delta_{k}
$$

(9) is the observation equation.

In order to do Kalman filter, $X_{k}$ is looked as the state vector to contain the dynamic noise, we have

$$
X_{k+1}=X_{k}+\Omega_{k}
$$

(10) can be written as

$$
X_{k+1}=\Phi_{k+1, k} X_{k}+\Omega_{k}
$$

Where $\Phi_{k+1, k}$ is a unit matrix. be

On the basis of (9) and (11), the state equation and observation equation of Kalman filter model can 
Obtained.

$$
\begin{gathered}
X_{k+1}=\Phi_{k+1, k} X_{k}+\Omega_{k} \\
L_{k+1}=B_{k+1} X_{k+1}+\Delta_{k+1}
\end{gathered}
$$

On the basis of (5), (6), (7), (12),(13), filter values of $a$ and $b$ can be obtained, fitting values of Kalman filter model can be obtained, and fitting values of settlement amounts can be obtained.

\section{Example of the calculation}

The settlement data of the monitoring point JD6 of the building are calculated, on the basis of the settlement data, $D_{\Delta}(k)= \pm 1 \mathrm{~mm}$.

Let $X(0 / 0)=\left[\begin{array}{l}0.51351 \\ 0.02669\end{array}\right]$, where 0.51351 and 0.02669 are calculated values of parameter $a$ and parameter $b$ in the hyperbolic curve model, the initial value of the state vector and dynamic noise are regarded as irrelevance, we have

$$
D_{X}(0)=D_{X}(0 / 0)=\left[\begin{array}{ll}
1 & 0 \\
0 & 1
\end{array}\right], D_{\Omega}(k)=\left[\begin{array}{ll}
1 & 0 \\
0 & 1
\end{array}\right] \text {, some computed results are listed in Table } 1 .
$$

Table 1. Settlement observation values and their computed results of the monitoring point JD6 of the building

\begin{tabular}{cccccc}
\hline $\begin{array}{c}\text { Observation } \\
\text { time } \\
\text { (year-month) }\end{array}$ & $\begin{array}{c}\text { Observation } \\
\text { values } \\
(\mathrm{mm})\end{array}$ & $\begin{array}{c}\text { Fitting values } \\
\text { of model } 1 \\
(\mathrm{~mm})\end{array}$ & $\begin{array}{c}\text { Residual errors } \\
\text { of model 1 } \\
(\mathrm{mm})\end{array}$ & $\begin{array}{c}\text { Fitting values } \\
\text { of model 2 } \\
(\mathrm{mm})\end{array}$ & $\begin{array}{c}\text { Residual errors } \\
\text { of model 2 } \\
(\mathrm{mm})\end{array}$ \\
\hline $2016-2$ & 12.22 & 11.831 & -0.389 & 12.130 & -0.090 \\
$2016-3$ & 13.78 & 13.508 & -0.272 & 13.839 & 0.059 \\
$2016-4$ & 14.11 & 15.034 & 0.924 & 14.192 & 0.082 \\
$2016-5$ & 16.34 & 16.429 & 0.089 & 16.668 & 0.328 \\
$2016-6$ & 17.65 & 17.708 & 0.058 & 17.640 & -0.010 \\
$2016-7$ & 18.12 & 18.886 & 0.766 & 18.134 & 0.014 \\
$2016-8$ & 20.33 & 19.975 & -0.355 & 20.301 & -0.029 \\
$2016-9$ & 21.01 & 20.983 & -0.027 & 21.014 & 0.004 \\
$2016-10$ & 22.17 & 21.920 & -0.250 & 22.166 & -0.004 \\
$2016-11$ & 23.38 & 22.793 & -0.587 & 23.376 & -0.004 \\
\hline
\end{tabular}

Model 1 is hyperbolic curve model, model 2 is Kalman filter model based on hyperbolic curve model, residual errors mean that fitted values of models subtract observation values

Table 1 shows that residual errors of hyperbolic curve model are great, the greatest residual error is $0.924 \mathrm{~mm}$, the smallest residual error is $-0.027 \mathrm{~mm}$. Residual errors of Kalman filter model based on hyperbolic curve model are smaller than $0.33 \mathrm{~mm}$, and the greatest residual error is $0.328 \mathrm{~mm}$, the smallest residual error is $0.004 \mathrm{~mm}$, the fitting precision of Kalman filter model based on hyperbolic curve model is higher.

The forecasted settlement value of the monitoring point JD6 of the building of hyperbolic curve model is $23.608 \mathrm{~mm}$ on December, 2016, the settlement observation value on December, 2016 is $24.03 \mathrm{~mm}$, the forecast error is $-0.422 \mathrm{~mm}$, the forecast error is greater.

The forecasted settlement value of the monitoring point JD6 of the building of Kalman filter model based on hyperbolic curve model is $24.211 \mathrm{~mm}$ on December, 2016, the settlement observation value on December, 2016 is $24.03 \mathrm{~mm}$, the forecast error is $0.181 \mathrm{~mm}$, the forecast error is smaller, the forecast effect is better. 


\section{Conclusions}

The paper looked parameters of hyperbolic curve model as the state vector to erect Kalman filter model based on hyperbolic curve model, the model is used to forecast the settlement value of the building. The calculation result show that Kalman filter model based on hyperbolic curve model is good in the suitability, the model precision and deformation forecast precision is high, the forecast effect is better.

\section{Acknowledgements}

This work was financially supported by the state natural sciences foundation (No. 41172298), scientific research foundation for NASG key laboratory of land environment and disaster monitoring (No. LEDM2013B03 ).

\section{References}

[1] Dong Jianguo, Zhao Xihong: Foundation of High Rise Building-Theory and Practice about Coaction. (Tongji University Press, China 1997).

[2] Jin Xiaoguang, Li Xiaohong: General Adequate Grey Model of Modeling Forecasting of Slope Deformation. The Chinese Journal of Geological Hazard and Control Vol.12(2001), p.52-57

[3] Jiang Gang, Lin Lusheng, Liu Zhude, et al: Prediction Grey Model for Slope Displacement. Rock and Soil Mechanics Vol.21(2000), p.244-246

[4] Tang Tianguo, Wan Xing, Liu Haowu: Improved GM Model for Safety Monitoring of High Rock Slopes. Chinese Journal of Rock Mechanics and Engineering Vol. 24(2005), p. 307-312

[5] Huang Zhiquan, Cui Jiangli, Liu Handong: Chaotic Neural Network Method for Slope Stability Prediction. Chinese Journal of Rock Mechanics and Engineering Vol. 23(2004), p.3808-3812

[6] Lu Jinhu, Chen Yifeng, Zhang Suochun: Slope Displacement Forecast Based on Adaptive Neural Network. Systems Engineering - Theory and Practice Vol. 21(2001), p. 124-129

[7] Zheng Dongjian, Gu Chongshi, Wu Zhongru: Time Series Evolution Forecasting Model of Slope Deformation Based on Multiple Factors. Chinese Journal of Rock Mechanics and Engineering Vol.24(2005), p. 3180-3184

[8] Cui Xizhang, Yu Zongchou, Tao Benzao, et al: General Surveying Adjustment. ( Surveying and Mapping Press, China 1992)

[9] Lu Fumin, Li Jin: Application of Kalman Filter Model with the Time and Excavation Depth to Building Deformation Analysis. Bulletin of Surveying and Mapping Vol.32(2012), p. 59-61

[10] Zhou Letao, Huang Dingfa, Yuan Lin guo, et al: A Kalman Filtering Algorithm for Online Integer Ambiguity Resolution in Reference Station Network. Acta Geodaetica et Cartographica Sinica Vol.36(2007), p.37-42 